\title{
Prediction-aided precoding for multiuser massive MIMO null-space expansion
}

\author{
Kabuto Arai $^{1, \text { a) }}$, Kazuki Maruta ${ }^{2, \text { b) }}$, and Chang-Jun Ahn ${ }^{1}$ \\ ${ }^{1}$ Graduate School of Engineering, Chiba University, \\ 1-33 Yayoicho, Inage-ku, Chiba-shi, Chiba 263-8522, Japan \\ ${ }^{2}$ Academy for Super Smart Society, Tokyo Institute of Technology, \\ Tokyo 152-8552, Japan \\ a)kabuto.arai@chiba-u.jp \\ b) kazuki.maruta@m.ieice.org
}

\begin{abstract}
This letter proposes a new precoding design scheme to improve the null-space expansion (NSE) for multi-user massive MIMO systems. In NSE, steering many nulls based on past channel state information (CSI) significantly improves inter-user interference (IUI) suppression capability in a time-varying channel environment. However, extensive nullification consumes a part of the degree of freedom (DoF) resulting in the degradation of the desired signal gain. Our new proposal predicts the desired user's channel state and derives the precoding weight to compensate for that loss. Computer simulation verifies the improved signal-to-interference-plus-noise power ratio (SINR) performance.
\end{abstract}

Keywords: null-space expansion, multiuser MIMO, massive MIMO, timevarying channels, channel prediction

Classification: Wireless Communication Technologies

\section{References}

[1] K. Maruta and F. Falcone, "Massive MIMO systems: present and future," Electronics, vol. 9, no. 3, p. 385, Feb. 2020.

[2] H.P. Bui, Y. Ogawa, T. Nishimura, and T. Ohgane, "Performance evaluation of a multi-user MIMO system with prediction of time-varying indoor channels," IEEE Trans. Antennas Propag., vol. 61, no. 1, pp. 371-379, Jan. 2013.

[3] A. Duel-Hallen, "Fading channel prediction for mobile radio adaptive transmission systems," Proc. IEEE, vol. 95, no. 12, pp. 2299-2313, 2007.

[4] K.E. Baddour and N.C. Beaulieu, "Autoregressive modeling for fading channel simulation," IEEE Trans. Wireless Commun., vol. 4, no. 4, pp. 1650-1662, 2005.

[5] T. Iwakuni, K. Maruta, A. Ohta, Y. Shirato, T. Arai, and M. Iizuka, "Null-space expansion for multiuser massive MIMO inter-user interference suppression in time varying channels," IEICE Trans. Commun., vol. E100-B, no. 5, pp. 865873, 2017.

[6] K. Arai, K. Maruta, and C-J. Ahn, "High beamforming gain precoding weight design scheme with null-space expansion for multiuser massive MIMO in timevarying channels," Proc. 23rd International Symposium on Wireless Personal Multimedia Communications (WPMC 2020), Oct. 2020. 
[7] Q.H. Spencer, A.L. Swindlehurst, and M. Haardt, "Zero-forcing methods for downlink spatial multiplexing in multiuser mimo channels," IEEE Trans. Signal Process., vol. 52, no. 2, pp. 461-471, 2004. DOI: 10.1109/wpmc50192.2020. 9309504

\section{Introduction}

Due to the explosive increase in the number of communication terminals, a high communication capacity is required. Massive multiple-input multiple-output (MIMO) system is still a key technology to meet the requirements of $5 \mathrm{G}$ and beyond mobile communication systems [1]. In the massive MIMO system, the base station (BS) is equipped with a huge number of antennas and can obtain significant spatial diversity. Multiuser MIMO supports multiple streams transmission for each user equipment (UE) simultaneously and at the same frequency. It is attained by beamforming/nullification techniques to separate them spatially by suppressing inter-user interference (IUI). At the BS side, channel state information (CSI) is required to suppress IUI. In the downlink trainsmission, CSI is estimated through uplink training or CSI feedback. If UEs move, the channel varies with time and the estimated CSI becomes outdated. As a result, IUI cannot be sufficiently suppressed, leading to a degradation in system capacity [2]. A channel prediction approach such as the autoregressive (AR) model has been proposed to compensate for channel time fluctuations [3,4]. It is useful but has limitations on IUI suppression capability because only one null can be steered per UE even with the predicted channel. To overcome this problem, the null-space expansion (NSE) scheme has been previously proposed [5]. It fully takes advantage of the huge degrees of freedom (DoF) in massive MIMO to steer additional nulls for further IUI suppression. However, in the NSE scheme, a part of DoF originally consumed for obtaining the desired signal gain is diverted to the additional null steering, so that the desired signal power is decreased. We newly propose a multiuser MIMO precoding that can maintain high beamforming gain with tracking time-varying channels predicted by the AR model in addition to the excellent interference suppression capability. This letter provides more extensive results with more stringent mobility parameters than our conference publication [6].

\section{Preliminaries}

\subsection{System model}

We consider a time-varying multiuser massive MIMO downlink system. BS is equipped with $N_{\mathrm{BS}}$ antennas, and $N_{\mathrm{UE}}$ single-antenna UEs are randomly moving. The channel matrix, $\mathbf{H}(t) \in \mathbb{C}^{N_{\mathrm{UE}} \times N_{\mathrm{BS}}}$ is represented as $\mathbf{H}(t)=\left[\mathbf{h}_{1}(t) \cdots \mathbf{h}_{N_{\mathrm{UE}}}(t)\right]^{T}$ where $\mathbf{h}_{k}(t)=\left[\begin{array}{lllll}h_{k 1}(t) & \cdots & h_{k j}(t) & \cdots & h_{k N_{\mathrm{BS}}}(t)\end{array}\right]^{T} \in \mathbb{C}^{N_{\mathrm{BS}} \times 1}$ represents the channel vector for the $k$-th UE, and $h_{k j}(t)$ is the channel coefficient between the $k$-th $\left(k=1,2, \ldots, N_{\mathrm{UE}}\right) \mathrm{UE}$ and $j$-th $\left(j=1,2, \ldots, N_{\mathrm{BS}}\right) \mathrm{BS}$ antenna. Based on the uplink pilot signal or CSI feedback from UEs, channel matrices $\left\{\mathbf{H}(0), \mathbf{H}\left(T_{\mathrm{f}}\right), \mathbf{H}\left(2 T_{\mathrm{f}}\right), \ldots\right\}$ 
are estimated in $T_{\mathrm{f}}[\mathrm{s}]$ intervals. Then, BS design the MIMO precoding weight $\mathbf{W}=\left[\begin{array}{lllll}\mathbf{w}_{1} & \cdots & \mathbf{w}_{j} & \cdots & \mathbf{w}_{N_{\mathrm{UE}}}\end{array}\right] \in \mathbb{C}^{N_{\mathrm{BS}} \times N_{\mathrm{UE}}}$ using estimated channel matrices.

\subsection{Null-space expansion (NSE)}

NSE steers many nulls to one UE, which provides excellent IUI suppression capability in time-varying channel environments [5]. Multiple nullification can be attained by exploiting the past $N_{\mathrm{f}}$ CSI estimates $\left\{\mathbf{H}\left(t_{0}\right), \mathbf{H}\left(t_{0}-T_{\mathrm{f}}\right), \ldots, \mathbf{H}\left(t_{0}-\left(N_{\mathrm{f}}-1\right) T_{\mathrm{f}}\right)\right\}$ where $t_{0}$ is the latest channel estimation timing. The NSE weight vector for the $k$-th $\mathrm{UE} \mathbf{w}_{k} \in \mathbb{C}^{N_{\mathrm{BS}} \times 1}$ is designed as

$$
\begin{aligned}
& \mathbf{h}_{k}^{T}\left(t_{0}\right) \mathbf{w}_{k}=1 \\
& \mathbf{H}_{\bar{k}}\left(t_{0}-n T_{\mathrm{f}}\right) \mathbf{w}_{k}=\mathbf{0}_{\left(N_{\mathrm{UE}}-1\right) \times 1} \\
& \text { for } n=0,1, \ldots, N_{\mathrm{f}}-1 \text { and } k=1,2, \ldots, N_{\mathrm{UE}}
\end{aligned}
$$

where $\mathbf{H}_{\bar{k}}(t)=\left[\mathbf{h}_{1}(t) \cdots \mathbf{h}_{k-1}(t) \mathbf{h}_{k+1}(t) \cdots \mathbf{h}_{N_{\mathrm{UE}}}(t)\right]^{T} \in \mathbb{C}^{\left(N_{\mathrm{UE}}-1\right) \times N_{\mathrm{BS}}}$. Eq. (1) indicates the beamforming for the $k$-th desired UE, and Eq. (2) represents the nullsteering for suppressing IUI from UEs except for the $k$-th one. From Eq. (2), we expect a high interference suppression effect since multiple nulls are steered for each UE including its future channel state. However, the desired signal gain decreases since some of the DoFs are consumed by enhanced null-steering. Besides, the signal gain also decreases with time since the latest estimated CSI $\mathbf{h}_{k}\left(t_{0}\right)$ in Eq. (1) becomes outdated.

\subsection{Channel prediction based on AR model}

AR model is widely used for predicting discrete time series [3]. Using the past $p$ estimated channels $\left\{h_{k j}\left(t_{0}\right), h_{k j}\left(t_{0}-1\right), \ldots, h_{k j}\left(t_{0}-(p-1) T_{\mathrm{f}}\right\}\right.$, the predicted channel one step ahead from the latest time instant $t_{0}, \hat{h}_{k j}\left(t_{0}+T_{\mathrm{f}}\right)$ is given as

$$
\hat{h}_{k j}\left(t_{0}+T_{\mathrm{f}}\right)=\sum_{n=0}^{p-1} a_{n}^{k} h_{k j}\left(t_{0}-n T_{\mathrm{f}}\right)
$$

where $p$ is order of the AR model, and $\left\{a_{0}^{k}, a_{1}^{k}, \ldots, a_{p-1}^{k}\right\}$ indicate AR coefficient for the $k$-th UE. AR coefficients satisfy the Yule-Walker equation [4] as

$$
\mathbf{R}_{k}=\left[\begin{array}{cccc}
\mathbf{R}_{k} \mathbf{a}_{k}=\mathbf{v}_{k} \\
R_{k}[0] & R_{k}^{*}[1] & \cdots & R_{k}^{*}[p-1] \\
\vdots & R_{k}[0] & \cdots & R_{k}^{*}[p-2] \\
R_{k}[p-1] & R_{k}[p-2] & \ddots & \vdots \\
R_{k}[0]
\end{array}\right], \mathbf{a}_{k}=\left[\begin{array}{c}
a_{0}^{k} \\
a_{1}^{k} \\
\vdots \\
a_{p-1}^{k}
\end{array}\right], \mathbf{v}_{k}=\left[\begin{array}{c}
R_{k}[1] \\
R_{k}[2] \\
\vdots \\
R_{k}[p]
\end{array}\right]
$$

where $R_{k}[m]$ is an autocorrelation function $(\mathrm{ACF})$ defined as

$$
R_{k}[m]=\mathrm{E}\left[h_{k j}(t) h_{k j}^{*}\left(t-m T_{\mathrm{f}}\right)\right] .
$$

Assuming the Jakes'model, $R_{k}[m]$ is given as $R_{k}[m]=J_{0}\left(2 \pi f_{\mathrm{d}} m T_{\mathrm{f}}\right)$ where $J_{0}(\cdot)$ is the Bessel function of the first kind of order zero and $f_{\mathrm{d}}$ is the maximum Doppler 


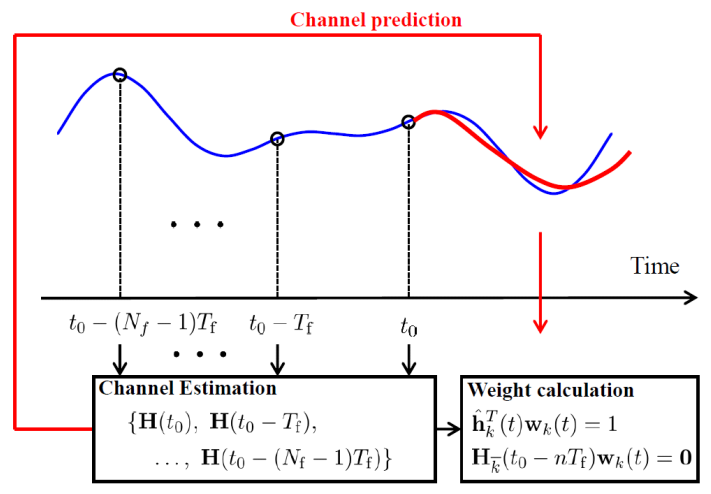

Fig. 1. The flow of the precoding weight design in the proposed scheme.

frequency. In practice, we cannot know maximum Doppler frequency $f_{\mathrm{d}}$. Thus we approximately compute ACF by past $N_{\mathrm{f}}$ observations as

$$
\hat{R}_{k}[m]=\frac{1}{N_{\mathrm{BS}}\left(N_{\mathrm{f}}-m\right)} \sum_{j=1}^{N_{\mathrm{BS}}} \sum_{n=0}^{N_{\mathrm{f}}-m-1}\left\{h_{k j}\left(t_{0}-n T_{\mathrm{f}}\right) h_{k j}^{*}\left(t_{0}-(n+m) T_{\mathrm{f}}\right)\right\} .
$$

From Eqs. (4) and (5), we can determine the AR coefficients as $\mathbf{a}_{k}=\hat{\mathbf{R}}_{k}^{-1} \hat{\mathbf{v}}_{k}$.

\section{Proposed scheme}

To compensate for the loss of desired signal gain in the NSE, the precoding weight vector is updated sequentially so as to track the continuously varying channels. Fig. 1 illustrates the proposed weight design flow. Using the past discretely estimated $N_{\mathrm{f}}$ channels $\left\{h_{k j}\left(t_{0}\right), h_{k j}\left(t_{0}-1\right), \ldots, h_{k j}\left(t_{0}-\left(N_{\mathrm{f}}-1\right) T_{\mathrm{f}}\right\}\right.$ and the predicted channel $\hat{h}_{k j}\left(t_{0}+T_{\mathrm{f}}\right)$ in Eq. (3), we can predict continuous time-varying channels $\hat{h}_{k j}(t)$ $\left(t_{0} \leq t<t_{0}+T_{\mathrm{f}}\right)$ by sinc interpolation as

$$
\hat{h}_{k j}(t)=\sum_{n=0}^{N_{\mathrm{f}}-1} h_{k j}\left(t_{0}-n T_{\mathrm{f}}\right) \frac{\sin \left(\pi\left(t-n T_{\mathrm{f}}\right) / T_{\mathrm{f}}\right)}{\pi\left(t-n T_{\mathrm{f}}\right) / T_{\mathrm{f}}}+\hat{h}_{k j}\left(t_{0}+T_{\mathrm{f}}\right) \frac{\sin \left(\pi\left(t+T_{\mathrm{f}}\right) / T_{\mathrm{f}}\right)}{\pi\left(t+T_{\mathrm{f}}\right) / T_{\mathrm{f}}} .
$$

Then, the proposed weight vector for the $k$-th $\mathrm{UE} \mathbf{w}_{k}(t)$ can be designed as

$$
\begin{aligned}
& \hat{\mathbf{h}}_{k}^{T}(t) \mathbf{w}_{k}(t)=1 \\
& \mathbf{H}_{\bar{k}}\left(t_{0}-n T_{\mathrm{f}}\right) \mathbf{w}_{k}(t)=\mathbf{0}_{\left(N_{\mathrm{UE}}-1\right) \times 1} \\
& t_{0} \leq t<t_{0}+T_{\mathrm{f}} \\
& \text { for } n=0,1, \ldots, N_{\mathrm{f}}-1 \text { and } k=1,2, \ldots, N_{\mathrm{UE}}
\end{aligned}
$$

where $\hat{\mathbf{h}}_{k}(t)=\left[\begin{array}{lllll}\hat{h}_{k 1}(t) & \cdots & \hat{h}_{k j}(t) & \cdots & \hat{h}_{k N_{\mathrm{BS}}}(t)\end{array}\right] . \quad$ As shown in Eq. (7), the proposed weight is updated sequentially according to the continuously predicted channel. Therefore, improved beamforming gain is expected even in time-varying environments. Besides, as shown in Eq. (8), the proposed weight steers many nulls to one UE as well as the NSE weight in Eq. (2). Thus significant IUI suppression capability can also be maintained. Note that it is not practical to update the weight vector completely continuously in Eq. (7). It is enough to update the weight vector for every symbol duration as long as that is smaller than the channel coherence time. 


\section{Computer simulation}

Here we verify the effectiveness of the proposed scheme by Monte Carlo simulations in which 100 time-varying channel realizations are considered. Evaluation metrics are: averaged signal-to-interference-plus-noise power ratio (SINR), interference signal power, and desired signal power at the UEs side. The BS has $N_{\mathrm{BS}}=100$ antenna elements. $N_{\mathrm{UE}}=5$ UEs move to arbitrary directions at a constant speed. We consider independent identically distributed (i.i.d.) Rayleigh channels generated by the Jakes' model. Normalized Doppler shift $f_{\mathrm{d}} T_{\mathrm{f}}$ is 0.3 , which indicates the intensity of channel time variation. $N_{\mathrm{f}}=12$ past estimated CSI are used. AR model order $p$ is calculated based on the Akaike Information Criterion (AIC). The downlink signalto-noise ratio (SNR) at a UE side is $30 \mathrm{~dB}$ in SISO (Single-Input Single-Output) case. The uplink SNR at the BS side, which means channel estimation error, is set to $40 \mathrm{~dB}$. We compare the performances of the four schemes listed below. (a) Prop.: the proposed scheme, (b) NSE: the conventional NSE, (c) ZF-AR: Zero-Forcing precoding [7] using the prediction channel based on AR model in Eq. (3), (d) ZF: Zero-Forcing precoding (without compensation for time-varying channel impact).

Figs. 2(a), 2(b), 2(c) shows the time variation of SINR, interference power, and desired power, respectively. The desired signal power is normalized to $0 \mathrm{~dB}$ in the SISO case. From Fig. 2(a), we see that the SINR performance of ZF degrades rapidly with time progress because it has no compensation capability for the time-varying channel. Prop., NSE, and ZF-AR have less degradation of SINR than the simple $\mathrm{ZF}$ thanks to their additional compensation function for the time-varying impact. Especially, the proposed scheme has the best SINR performance among these four candidates. As shown in Fig. 2(b), our proposal and the conventional NSE can suppress interference significantly since they perform additional nullification for interfering UEs. As shown in Fig. 2(c), the desired power of NSE degrades drastically since the beamforming weight in Eq. (1) is always fixed. The proposed scheme has a smaller degradation of the desired power than NSE, because the proposed weight is designed to follow the time-varying channel as formulated in Eq. (7). Meanwhile, the desired signal power of the NSE-based schemes, including our proposal, is less than ZF-AR since they consume DoFs for extensive null-steering. Table I summarizes the time-averaged SINR, interference signal power, and desired signal power. Our proposed scheme improves the desired power by about $1.9 \mathrm{~dB}$ compared with the conventional NSE while keeping outstanding interference suppression capability. As a result, the SINR of the proposed scheme is improved by about $2.0 \mathrm{~dB}$. To verify the effective region of the proposed method, Figs. 2(d) and 2(e) presents SINR versus uplink SNR and normalized Doppler shift $f_{\mathrm{d}} T_{\mathrm{f}}$, respectively. We see that the proposed scheme works effectively in the region where uplink SNR is larger than $25 \mathrm{~dB}$ and $f_{\mathrm{d}} T_{\mathrm{f}}$ is less than 0.45 . Nevertheless, the proposed scheme attains almost the best performance. We can conclude that our overall approach could be the most powerful solution to realize a stable multiuser MIMO transmission in the mobility environment. 


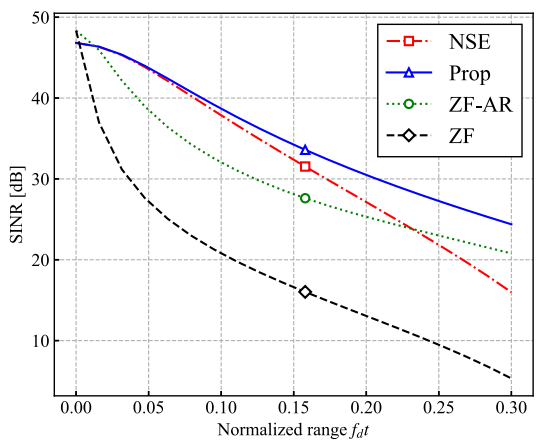

(a) Time variation of SINR.

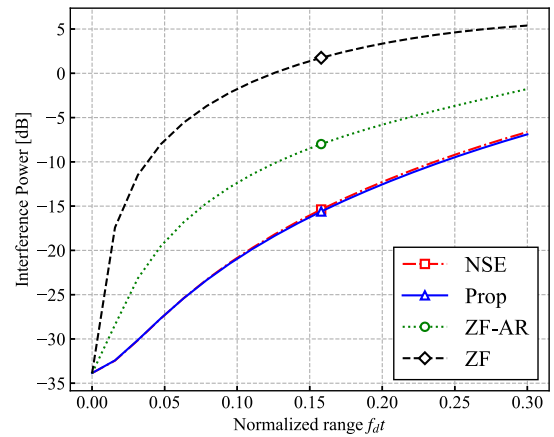

(b) Time variation of interference power.

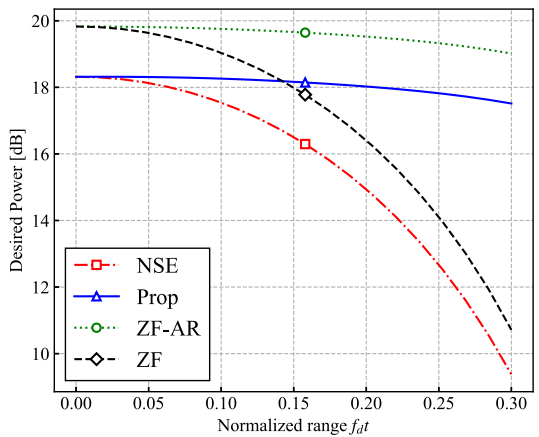

(c) Time variation of desired power.

Fig. 2. Simulation results $\left(f_{\mathrm{d}} T_{\mathrm{f}}=0.3\right.$, downlink $\mathrm{SNR}=$ $30 \mathrm{~dB})$

Table I. Time-averaged SINR, interference power, and desired power.

\begin{tabular}{cccc}
\hline Method & SINR $[\mathrm{dB}]$ & Interference power $[\mathrm{dB}]$ & Desired power $[\mathrm{dB}]$ \\
\hline Prop & 30.8 & -12.8 & 18.1 \\
NSE & 28.8 & -12.6 & 16.2 \\
ZF-AR & 26.4 & -6.80 & 19.6 \\
ZF & 16.0 & 1.78 & 17.6 \\
\hline
\end{tabular}

\section{Conclusion}

This letter proposed a predicted-aided precoding weight design with NSE for multiuser massive MIMO transmission in time-varying channels. It can prevent loss of the desired signal gain by channel prediction on the AR model while keeping an ex-

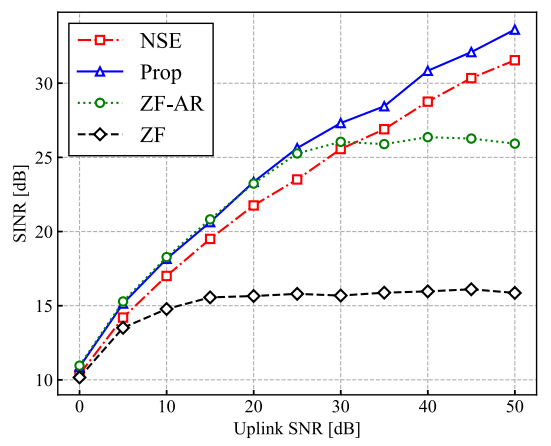

(d) SINR versus uplink SNR.

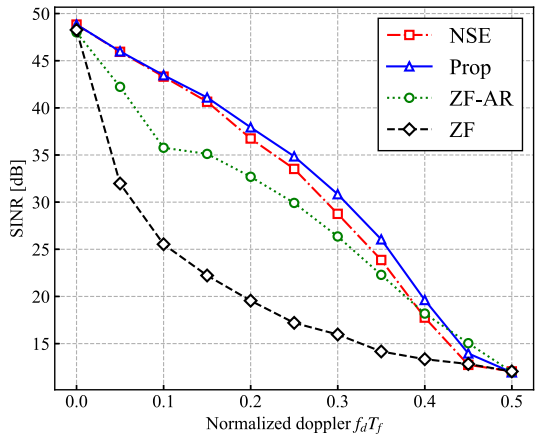

(e) SINR versus normalized Doppler shift $f_{\mathrm{d}} T_{\mathrm{f}}$. 
cellent interference suppression capability provided by NSE. Computer simulations confirmed improvements of the desired power and SINR by about $1.9 \mathrm{~dB}$ and $2.0 \mathrm{~dB}$ compared with the conventional NSE method, respectively.

\section{Acknowledgments}

This work was supported by the KDDI Foundation, the Mazda Foundation, and KAKENHI Grant-in-Aid for Scientific Research (B) (20H04178). 